\title{
Inverse Transmission Line Modeling of GPR for Landmine Detection and Subsurface Parameter Estimation
}

\author{
Gebremichael Teame Tesfamariam*
}

School of Electrical and Computer Engineering, Ethiopian Institute of Technology-Mekelle (EiT-M), P.O. Box 231, Mekelle University, Mekelle, Ethiopia (*gtesfa@mu.edu.et).

\begin{abstract}
This paper presents detection of plastic landmines and characterization of subsurface and the target using ground penetrating radar (GPR) measurements. The inverse transmission line (TL) modeling approach is used to analyze the characteristics of practical GPR system operating above non-uniform ground. The modeling approach considers the presence of surface roughness, natural clutter, targets and soil moisture. With this model, the time domain signature of electromagnetic (EM) propagation can be assessed. The inverse TL modeling is applied to estimate the characteristic parameters (such as conductivity, magnetic permeability and permittivity) of the subsurface and buried plastic landmines. TL modeling is applied to obtain the reverse solution of the electromagnetic equations. Different scenarios were considered and test signals of the B-scan data are used to test the effectiveness of the method. Simulations of the real data analysis showed the effectiveness of the model and its application to target detection and characterization of the subsurface. Performance of the modeling is analyzed using receiver operating characteristic (ROC) analysis of the four hypotheses and it has found that $99.5 \%$ detection has achieved as the cost of $12 \%$ of false alarm.
\end{abstract}

Keywords: Dielectric properties; EM wave propagation; GPR; Parameter estimation; Inverse transmission line.

\section{INTRODUCTION}

Subsurface characterization is important for the study of the nature of underground structures, utility lines, drainage lines and other objects buried in the ground. Landmines, anti-personal and antitank, are devices planted during conflict in the subsurface or thrown on the surface to injure (kill or maim) enemy forces. Decades later, they injure indiscriminately whoever (civilians or animals) who triggers as explained in MAction (2015), MAction (2014), MAction (2011), MAction (2010), MAction (2009). According to the study by Zoubir et al. (2001), Daniels (2009), MAction (2010); and MAction (2009), hundreds of millions of landmines actively lodged globally due to many international wars and internal conflicts, are causing causalities to innocent civilians and animals.

The modern landmines are mainly comprised of nonmetallic substances with wood, ceramic or plastic casings. The magnetic properties of plastics, ceramics and wood are similar to the ground 
surface. Conventional de-mining techniques, such as metal detector, discriminate landmines based on the metallic contents of the landmine. Zoubir et al. (2001); and Gebremichael (2013) explained that the classical de-mining techniques used for the detection of modern landmines are slow, difficult and expensive.

Ground penetration radar (GPR) system is one of the techniques used to detect the subsurface objects. It provides a centimeter resolution to locate small objects with no or low metallic contents (Zoubir et al., 2001). The system is makes use of non-destructive device that do not affect the environment and the targets (Xu, 2002). There are also shortcomings in the GPR system performance due to the presence of strong noise, clutter and subsurface roughness (Zoubir et al., 2001). However, detection performance of GPR can be improved with the application of advanced signal processing techniques which does filtering and shaping of the received signal.

The GPR system transmits an EM pulse trains based on speed or displacement of the system. The pulse travels through the ground gets reflected at layer boundaries and is received by the GPR system. The transmitted wave propagates at a velocity dependent on the dielectric constant of the media. Reflections are caused due to the dielectric contrast of the adjacent media. The travel time of the pulse is related to the depth from which the energy is reflected. Thus, interpretation of the reflected energy provides information on structural variation of the subsurface, and presence of buried anomalies.

Transmission line (TL) modeling is a conceptually simple approach, which can be used to gain an insight into the operation of GPR (Daniels, 2004; Jol, 2010; Lipiniski, 2008). In TL modeling, the subsurface ground and the buried targets are considered as layers of small sections of lumped transmission line cascaded vertically (Gebremichael, 2013) (see Fig 1). The model generates a set of reflections, scaled in terms of amplitude and time, and convolved with the driving impulse function. In the course of calculating the reflections, multi-reflections at the boundaries of a given medium are also taken into account (Gebremichael, 2013). Landmines in the subsurface are considered as layers of media having distinct electrical properties.

Non-statistical modeling of GPR responses (via finite-difference time-domain (FDTD) modeling and time-frequency analysis, for example) is widely explored field, and significant advances have made it possible to accurately model target responses under heterogeneous and realistic soils. Despite these advances, the computational complexity of most electromagnetic modeling 
techniques coupled with the unknown composition of soils prohibit the real-time application of these models in fielded scenarios. Moreover, inverse modeling of these techniques doesn't provide sufficient information about target detection.

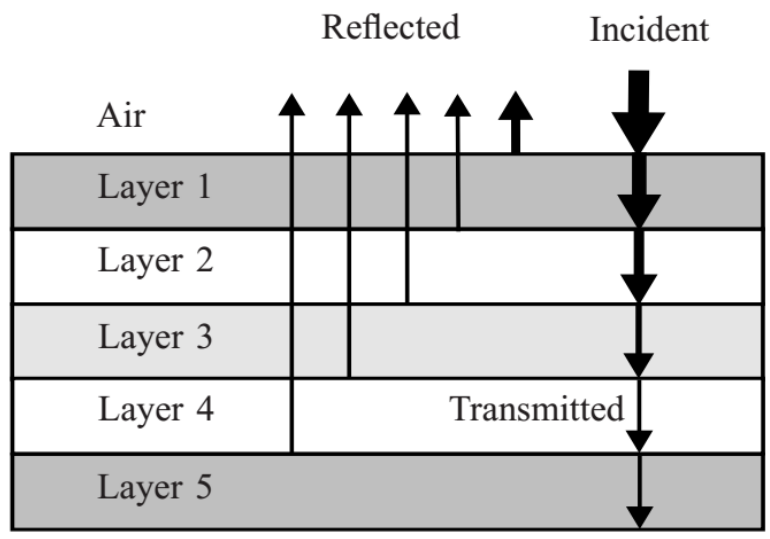

Figure 1. Layout of transmission line model (Gebremichael, 2013).

Many authors are conducting GPR -based landmine detection researches. Among them, Zoubir et al. (2001) have considered many signal processing techniques and found that the Kalman filter based approach is the most powerful. Gebremichael (2013) also considered many signal processing techniques and found that symmetry filtering and adaptive background subtraction is very effective. Barakat et al. (2000) have considered time-frequency techniques for the detection of plastic landmines. However, symmetry filtering considers the object to be symmetrical. Most of the above techniques are not valid or effective because they are based on offline processing. However, landmines demand an online detection.

In this paper, the inverse TL modeling approach is used to analyze real GPR data, based on the surface reflection parameter method (SRPM) (Gebremichael, 2013; GSSI, 2005; and Arunachalam et al., 2006). In this approach, reflected peak layer voltage amplitudes and arrival time were measured. These measurements are compared to the incident voltage and a signal modeled using TL modeling, to calculate the reflection coefficients, intrinsic impedance and relative permittivity (Lambot et al., 2004; Liu et al., 2002). Each layer is assumed as lossless, where the conductivity $\sigma_{n}=0$, in the estimation of the relative permittivity of the layer.

This paper tries to estimate the electromagnetic properties of the subsurface and buried anomalies. The estimated parameters are used to detect the presence of landmines and classify it from the background clutter. A-scans, reflection of a single pulse from the measured GPR data, 
are considered and the consecutive peaks of the A-scan indicate the reflections from the interfaces of the layers.

\section{TL MODELING PRINCIPLES}

The A-scan signal received from GPR is wavelet shaped and at horizontal position $x$ and discrete time instants, $t=1, \cdots, \mathrm{N}$, is given by the following (Gebremichael, 2013; Valle et al., 2000):

$$
Z(t, x)=W_{0}(t)+\sum_{m=-M}^{M} W(m) F(t-m, x)+N(t, x)
$$

Where, $W(m)$ is a driving wavelet function of width $2 M+1, W_{0}$ is direct pulse measured by the receiver antenna as crosstalk, $N(t, x)$ is additive noise measured at the receiver antenna and $F(t, x)$ represents the magnitude of the pulses reflected at the boundaries, and is given by:

$$
F(t, x)=\sum_{n=0}^{n_{x}-1} P_{t r}(n, x) \delta\left(t-t_{n}(x)\right)
$$

Where, $n_{x}$ is the maximum number of layers identified at a given antenna position $\mathrm{x}, P_{t r}$ is the transmission and reflection product of the $\mathrm{n}^{\text {th }}$ layer, $t_{n}$ is layer's calculated equivalent travel time and $\delta(\mathrm{t})$ is the Kronecker delta function, considered as pulse signal of GPR system.

\subsection{EM Wave Propagation in Dielectric Materials}

When an EM wave propagates in a lossy dielectric media in $\mathrm{z}$ (downward)-direction, the electric field component, $E$, in X-direction is given below (Sadiku, 2010, and Maurice, 2010):

$$
E_{x}(z, t)=\operatorname{Re}\left(E_{0} e^{-\gamma z} e^{j \omega t}\right)=E_{0} e^{-\alpha z} \cos (\omega t-\beta z)
$$

Where, Re indicates the real component. Similarly, the magnetic field intensity component, $\mathrm{H}$, is given as (Sadiku, 2010)

$$
H_{y}(z, t)=\operatorname{Re}\left(H_{0} e^{-\gamma z} e^{j \omega t}\right)=H_{0} e^{-\alpha z} \cos (\omega t-\beta z)
$$

Where, $H_{0}=\frac{E_{0}}{\eta}$, and $\eta$ is a complex quantity known as the intrinsic impedance of the medium (in Ohms); $\alpha, \beta$ and $\gamma$ are the attenuation, phase and complex propagation constants respectively and $\omega$ is the angular frequency of the electromagnetic wave in $(\mathrm{rad} / \mathrm{sec})$. The propagation constant, $\gamma$, is given by:

$$
\gamma=\alpha+j \beta=\sqrt{j \omega \mu(\sigma+j \omega \varepsilon)}
$$


The propagation velocity, $v$, of an EM wave in a medium is given by

$$
v=\frac{c}{\left(\frac{\mu_{r} \varepsilon_{r}}{2}\left[\sqrt{1+\left(\frac{\sigma}{\omega}\right)^{2}}+1\right]\right)^{1 / 2}}
$$

Where, $c=3 \times 108 \mathrm{~m} / \mathrm{s}$ is velocity of EM waves in free space, $\varepsilon_{r}$ is relative permittivity, $\varepsilon$ is absolute permittivity, $\mu_{r}$ is relative permeability, $\mu$ is absolute permeability, and $\sigma$ is conductivity of the medium.

\subsection{Transmission Line Equations}

Transmission line (TL) is a pair of electrical conductors carrying an electrical signal from one place to another in a fixed time (Sadiku, 2010; and Chien et al., 2007). A lumped model of small length TL is given in figure 2. Using simple circuit analysis, the voltage and current equations of the lamped system are-

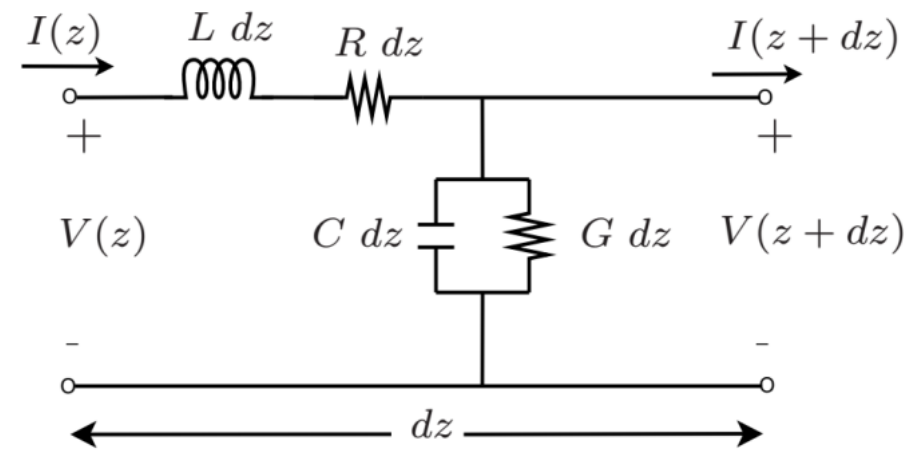

Figure 2. Lumped model of TL with vertical length of $d z$.

$$
\begin{gathered}
V(z)-V(z+d z)=\frac{\partial I(z)}{\partial t} L d z+I(z) R d z \\
I(z)-I(z+d z)=\frac{\partial V(z+d z)}{\partial t} C d z+V(z+d z) G d z
\end{gathered}
$$

Where, the lumped circuit parameters; $R, L, G$ and $C$ represent the resistance, inductance, conductance and capacitance of the element respectively.

The time harmonic analysis of this circuit gives the following wave equations,

$$
\frac{\partial V(z)}{\partial z}=(R+j \omega L) I(z) \quad \text { and } \quad \frac{\partial I(z)}{\partial z}=(G+j \omega C) V(z)
$$

Differentiating both equations of (9) and rearranging gives,

$$
\frac{\partial^{2} V(z)}{\partial z^{2}}-\gamma^{2} V(z)=0 \quad \text { and } \quad \frac{\partial^{2} I(z)}{\partial z^{2}}-\gamma^{2} I(z)=0
$$

(C) CNCS, Mekelle University 
The solutions for the wave equations (9) and (10) is given by:

$$
V(z, t)=V_{0} e^{-\gamma z} e^{j \omega t}=V_{0} e^{-\alpha z} \cos (\omega t-\beta z)
$$

Where, $\gamma=\sqrt{(R+j \omega L)(G+j \omega C)}$ is the propagation constant.

The EM wave propagation equations in dielectric materials are similar to the EM wave equations in TL. The electric field equation in (3) and the voltage equation (Jol, 2010) can be used interchangeably. Hence, the equivalence can be achieved using the assumptions, $V=E, L=\mu, C=\varepsilon, G=\sigma, R=0$ (lossless media) and $Z_{0}=\eta$.

The TL equivalent equation is a good model for the GPR EM wave propagation in the subsurface. This modeling approach can be used to assess the time domain signature of a physical situation. Each layer in the subsurface is modeled using equivalent impedance and propagation constants. Transmission and reflection coefficients are also calculated at the boundaries of any two media. Propagation velocity, equivalent travel time and the material losses are also included.

In this modeling approach, each layer will have attributes: range to layer boundary, $r_{n}$; conductivity, $\sigma_{n}$ or loss tangent, $\sigma_{n} /\left(\omega \varepsilon_{n}\right)$; relative dielectric constant, $\varepsilon_{r n}$; and relative magnetic permeability constant, $\mu_{r n}$. The absolute quantities are determined from the product of the relative values and the free space quantities, $\varepsilon_{n}=\varepsilon_{r n} \varepsilon_{0}$ and $\mu_{n}=\mu_{r n} \mu_{0}$. The free space quantities $\mu_{0}=4 \pi \times 10^{-7} \mathrm{H} / \mathrm{m}$ and $\varepsilon_{0}=8.85 \times 10^{-12} \mathrm{~F} / \mathrm{m}$.

Impedance of the lumped circuit in figure 2, is given by $\sqrt{(R+j \omega L)(G+j \omega C)}$. Using the above analogy, impedance of the $\mathrm{n}^{\text {th }}$ layer is given by:

$$
\eta_{n}=\sqrt{j \omega \mu_{n}\left(\sigma_{n}+j \omega \varepsilon_{n}\right)}
$$

At the $\mathrm{n}^{\text {th }}$ layer, reflection coefficient in the forward direction, when wave travels from $n^{\text {th }}$ to $(n+1)^{\text {th }}$ layer is given by (Gebremichael, 2013):

$$
\Gamma_{n}=\Gamma_{n-1, n}=\frac{\eta_{n}-\eta_{n-1}}{\eta_{n}+\eta_{n-1}}
$$

Similarly, the transmission coefficient in the forward direction is given by:

$$
\tau_{n}=\frac{2 \eta_{n-1}}{\eta_{n}+\eta_{n-1}}
$$

Signal reflected from each layer is represented by a delta function of time, transmission and reflection coefficients. The back scattered signal is calculated as a convolution sum of the delta (C) CNCS, Mekelle University 
function and the driving impulse function as in eq. 1. The equivalent travel time of a layer is given by

$$
\mathrm{t}_{n}=\frac{r_{n}-r_{n-1}}{v_{n}}
$$

Where, $v_{n}$ is the velocity of the EM wave in a given layer, for $\mu_{r n}=1, v=\frac{c}{\sqrt{\varepsilon_{r n}}}$.

Most antennas used in surface-penetrating applications have limited low frequency response and tend to act as high pass filters effectively differentiating the applied impulse, hence creating a wavelet (Gebremichael, 2013; and Jol, 2010). For this reason, a second order Ricker wavelet is used as a driving impulse function for the GPR antenna under consideration, and is given by:

$$
W_{2}(m)=A\left(1-2 \pi^{2} f_{c}^{2} m^{2}\right) e^{-\pi^{2} f_{c}^{2} m^{2}},-M \leq m \leq M
$$

Where, $A$ is gain of the impulse signal, $f_{c}$ is the center frequency of the radar antenna, $M$ is a positive integer and $M=5$ is commonly used in literature (Daniels, 2004).

\section{INVERSE MODEL BASED PARAMETER ESTIMATION}

In this section, SRPM technique is applied to estimate antenna height, ground and target parameters. For known pulse amplitude or transmitter antenna terminal voltage, the air-ground reflection coefficient is the ratio of the reflected peak voltage to the incident voltage (GSSI, 2005; and Helemi et al., 2008). However, from layers lower than the ground surface, the voltage ratio is given as a product of the reflection coefficient and the two-way transmission coefficients of the above layers (GSSI, 2005).

A reference A-scan signal $u_{m}(t)$ is modeled using the TL approach for an EM wave propagating from a height similar to the GPR antenna height. The subsurface is assumed to be target-free with average soil characteristics. This signal will serve as a reference signal for the parameter estimation of real GPR data. Mean square error between the real and estimated data can be used as a validation technique for the estimation.

From each measured A-scan, the mean value is subtracted to remove the low frequency DC offset component. The absolute peak amplitude $\left|E_{p l}\right|$ and arrival time $t_{p l}$ are measured and recorded in the vicinity of $0 \leq t \leq t^{*}+\tau / 2$, where $t^{*}$ is the absolute peak time of the modeled signal $u_{m}(t)$ and $\tau$ is the antenna pulse width. A shifting and scaling is introduced to the modeled signal so that the peak value and its peak time matches to the present A-scan. The shifted and 
scaled model signal is then subtracted from the measured A-scan to remove the first peak. Parameter estimation of the consecutive layers will follow the same procedure.

In this procedure we assume three subsurface layers that the first layer corresponds to the ground soil, the second layer corresponds to landmine (target) or mine-like objects and the third layer corresponds to soil subsurface which is assumed to have similar characteristics to the first layer. The first task in ground parameter estimation is to remove the DC offset and is given by:

$$
u(t, x)=A(t, x)-\frac{1}{N} \sum_{t=1}^{N} A(t, x)
$$

Where, $A(t, x)$ is measured A-scan at position $x, t=1,2,3 \cdots$, denotes two-way travel time at a given antenna position.

Reflection strength of each layer is measured as a ratio of the peak voltage reflected from each layer to the incident amplitude. Figure 3 shows a general shallow layer transmission-reflection scenario and the ratio of the reflected to the incident is given by equation 18 .

$$
\frac{\left|E_{p n}\right|}{\left|E_{i}\right|}=T_{0,1} T_{1,2} \ldots T_{n-1, n} \ldots T_{2,1} T_{1,0}
$$

Where, $\left|E_{p n}\right|$ s the absolute peak value of electric field reflected from the $\mathrm{n}^{\text {th }}$ layer, $\left|E_{i}\right|$ is the incident electric field, $T_{i, j}$ and $\Gamma_{i, j}$ are respectively the transmission and reflection coefficients at the interface of the $i-j$ layers.

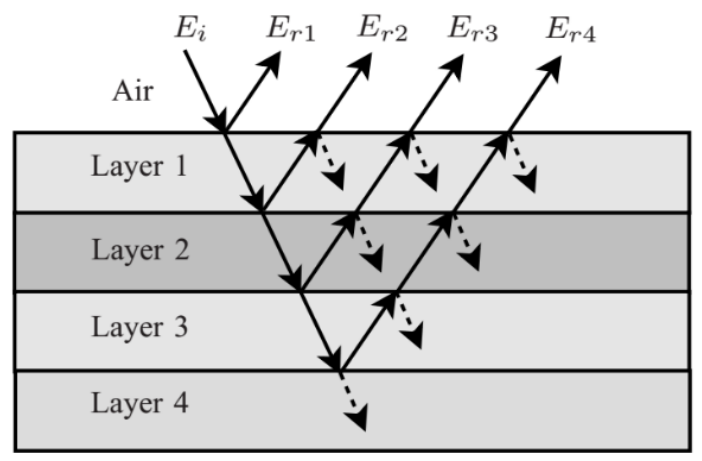

Figure 3. Reflections from lossless shallow subsurface layers (Helemi et al., 2008).

\subsection{Antenna Height Estimation}

The first subsurface reflection echoes are processed to provide estimations of the antenna height, roughness and reflection coefficient of the layer. Radargram of two targets, PMN1 and PMN2 under wet clay soil is given in figure 4. Radargram or B-scan is a two dimensional data 
composed of many A-scans at different positions and each A-scan has 512 sample points. In GPR measurement, the antenna should generally be placed close to the ground so as to reduce air-ground interface reflections for subsurface investigation. However, for landmine detection applications the antenna usually adjusted to scan 1.5 to $3 \mathrm{~cm}$ above the ground to avoid detonation of the surface lying landmines (Zoubir et al., 2001).

The antenna height $h_{a}$ is estimated as a product of the velocity of the EM wave in air and arrival time of the first peak. The arrival time of the first peak in the backscattered A-scan is half of the two way travel time, and is calculated as:

$$
t_{p 1}(x)=\underset{t}{\arg \max }|u(t, x)|, \quad 0 \leq t \leq t^{*}+\frac{\tau}{2}
$$

Where $\tau$ is the pulse width of the driving function, and $t^{*}$ is peak time of the modeled signal and $E_{p 1}(x)$, the first peak value of the A-scan, as shown in figure 5, is mathematically expressed as:

$$
E_{p 1}(x)=\left.u(t, x)\right|_{t=t_{p 1}}
$$

Since, the speed of an EM wave in air is the same as the speed of light in free space and the height of the antenna and antenna position $x$ is estimated as:

$$
h_{a}(x)=c \frac{t_{p 1}(x)}{2}
$$

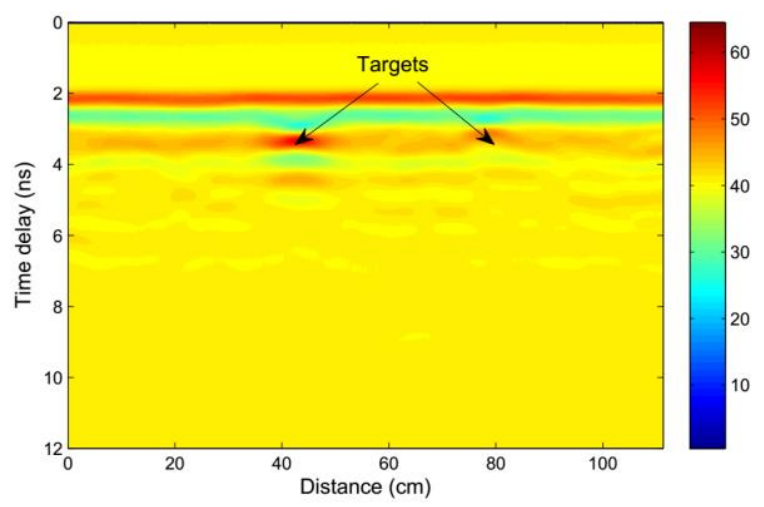

Figure 4. B-Scan (radargram) of PMN1 and PMN2 under wet clay soil.

\subsection{Soil Characteristic Estimation}

Assuming the incident wave is perpendicular to the surface and lossless media, the air-ground reflection coefficient is calculated as a ratio of the first peak of an A-scan to the incident amplitude (Lambot et al., 2004). The air-ground reflection coefficient is always negative and can be estimated using the voltage standing wave ratio (VSWR) of a transmission lines as: 


$$
\Gamma_{0,1}(x)=\frac{\eta_{1}(x)-\eta_{0}}{\eta_{1}(x)+\eta_{0}}=-\frac{E_{p 1}(x)}{\left|E_{i}\right|}
$$

Where, $E_{p 1}(x)$ is the first peak of measured A-scan, the intrinsic impedance of air $\eta$ ois known to be $\sqrt{\mu_{0} / \varepsilon_{0}}=376.82 \Omega$ and from (22), the intrinsic impedance of the first layer is calculated as

$$
\eta_{1}(x)=\eta_{0} \frac{1-\Gamma_{01}(x)}{1+\Gamma_{01}(x)}
$$

Assuming the ground surface is lossless, the relative permittivity of the ground surface can be estimated as a function of the reflection coefficient of the first layer as:

$$
\epsilon_{r 1}(x)=\left(\frac{1+\Gamma_{01}(x)}{1-\Gamma_{01}(x)}\right)^{2}
$$

\subsection{Target Characteristic Estimation}

The reflection coefficient, $\Gamma_{n, n-1,}$,represents the relationship between the impedances of the adjacent media reflected and incident energy of a plane wave. The reflection coefficient at the interface of free space and a different media, assuming a normally incident plane wave on a planar interface, the reflection coefficient is:

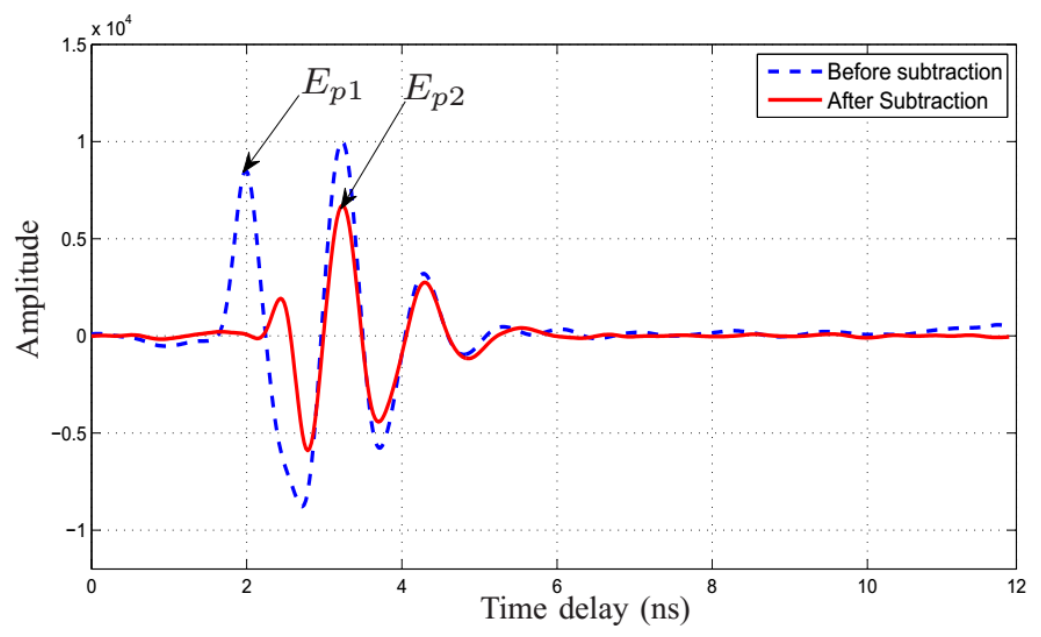

Figure 5. A-Scan, at $45 \mathrm{~cm}$, before and after subtraction of the first layer.

$$
\Gamma_{0,1}=\frac{\eta_{1}-\eta_{0}}{\eta_{1}+\eta_{0}}=\frac{1-\sqrt{\varepsilon_{1}}}{1+\sqrt{\varepsilon_{1}}}
$$

To estimate the second layer parameters, we subtract the scaled and shifted model signal to remove the first peak and search for the next peak voltage and arrival time are given as the maximum and argument of the maximum value of the difference of the incident and, the scaled and shifted signals respectively as: 


$$
\begin{gathered}
E_{p 2}(x)={ }_{t}^{\max }\left|u(t, x)-\alpha_{\text {ref }}(x) u_{m}\left(t-t_{\text {ref }}\right)\right| \\
t_{p 2}(x)=\underset{t}{\arg \max }\left|u(t, x)-\alpha_{r e f}(x) u_{m}\left(t-t_{\text {ref }}\right)\right|
\end{gathered}
$$

Where, $\alpha_{\text {ref }}(x)=E_{p}(x) / u_{m}\left(t^{*}\right) \mid$ is the scaling introduced at position $x, u_{m}(t)$ is modeled signal and $t_{r e f}(x)=t^{*}-t_{p l}(x)$ is time shift introduced by the A-scan measured at given antenna position. The thickness of the first layer which is the same as the depth of the target below the first surface is estimated as:

$$
d_{2}(x)=\frac{c}{2 \sqrt{\varepsilon_{1}(x)}}\left(t_{p 2}(x)-t_{p 1}(x)\right)
$$

To compute the relative permittivity of a second layer, the EM wave interaction of the first and second media need to be considered. The composite reflected signal from the second layer is the product of the incident, transmission coefficient of the first interface, the reflection coefficient of the second interface, and the transmission coefficient back through the first interface as given in the equation (18).

$$
\begin{gathered}
\frac{E_{p 2}(x)}{\left|E_{i}\right|}=T_{0,1}(x) \Gamma_{1,2}(x) T_{1,0}(x) \\
=\left(1+\frac{\eta_{1}(x)-\eta_{0}}{\eta_{1}(x)+\eta_{0}}\right) \Gamma_{1,2}(x)\left(\frac{\eta_{2}-\eta_{1}}{\eta_{2}+\eta_{1}}\right)\left(1+\frac{\eta_{0}-\eta_{1}(x)}{\eta_{0}+\eta_{1}(x)}\right) \\
=\left(\frac{4 \eta_{1}(x) \eta_{0}}{\left(\eta_{1}+\eta_{0}\right)^{2}}\right) \Gamma_{1,2}\left(\frac{\eta_{2}-\eta_{1}}{\eta_{2}+\eta_{1}}\right)
\end{gathered}
$$

In the interface of the first and second layers, the reflection coefficient, $\Gamma_{1,2}$, and impedance of the second layer, respectively, are given by the following.

$$
\begin{gathered}
\Gamma_{1,2}(x)=-\frac{\left|E_{i}\right|}{E_{p 2}(x)}=\frac{4 \eta_{0} \eta_{1}(x)}{\left(\eta_{1}(x)+\eta_{0}\right)^{2}} \\
\eta_{2}(x)=\eta_{1}(x)\left(\frac{4\left|E_{i}\right| \eta_{0} \eta_{1}(x)+E_{p 2}(x)\left(\eta_{1}(x)+\eta_{0}\right)^{2}}{4\left|E_{i}\right| \eta_{0} \eta_{1}(x)+E_{p 2}(x)\left(\eta_{1}(x)-\eta_{0}\right)^{2}}\right)
\end{gathered}
$$

Assuming the impedance in (30) is due to the lossless components, the relative permittivity of the second layer can be calculated from (29) and (30) as:

$$
\epsilon_{2}(x)=\varepsilon_{1}(x)\left(\frac{1+\Gamma_{12}(x)}{1-\Gamma_{12}(x)}\right)^{2}
$$




\section{HYPOTHESIS TESTING}

Reflection of an EM wave from an interface occurs only when adjacent media have different electromagnetic properties, especially different intrinsic impedances. The difference in permittivity of the adjacent media gives rise to difference in impedance, which causes an incident EM wave to reflect from the interface. Therefore, the hypotheses for testing the presence of a target could be defined easily based on one of the four parameters, namely, reflected peak voltage, reflection coefficient, impedance and relative permittivity.

Objective of this hypothesis is to test the presence of a target, that is, $H_{1}: E_{p 2}(x)>$ 0 against $H_{0}: E_{p 2}(x)=0$. Presence ofa target is declared if there is considerable reflection from the second layer or equivalently the impedance of the second layer is different from the first layer.

\subsection{Hypothesis Definition}

\subsubsection{Based on Peak Reflected Amplitude}

Here, the hypothesis is based on a test statistic, $T_{1}(x)=\max _{t}\left|u(t, x)-\alpha_{r e f}(x) u_{m}\left(t-t_{r e f}\right)\right|$. The null hypothesis (target free) is declared if the test statistic is smaller than some threshold value, $T(\alpha), H_{0}: T_{1}(x)<\epsilon_{1}$, otherwise, the alternative is accepted if, $H_{1}: T_{1}(x)>\epsilon_{1}$, where the threshold $T(\alpha)=\epsilon_{1} \geq 0$ is determined empirically.

$$
\begin{gathered}
H_{1} \\
T_{1}(x) \stackrel{\gtrless}{\gtrless} T(\alpha) \\
H_{0}
\end{gathered}
$$

\subsubsection{Based on Reflection Coefficient}

The test statistics in this case is defined as function of the reflection coefficient. The null hypothesis is accepted if there is no considerable reflection in the boundary of the first and second layers, $H_{0}: \eta_{2}(x)=\eta_{1}(x)$, otherwise the alternative is accepted if, $H_{1}: \eta_{2}(x) \neq \eta_{1}(x)$. The test statistic is defined as, $T_{2}(x)=\left|\Gamma_{12}(x)\right|$

$$
\begin{gathered}
H_{1} \\
T_{2}(x) \underset{\epsilon_{0}}{\gtrless} \epsilon_{2} \\
H_{0}
\end{gathered}
$$

\subsubsection{Based on Relative Permittivity}

The null hypothesis is accepted if the first and second layers have equal permittivities, $H_{0}: \varepsilon_{2}(n)=\varepsilon_{1}(n)$, otherwise the alternative is accepted if $H_{1}: \varepsilon_{2}(n) \neq \varepsilon_{1}(n)$. The test statistic in this case is $T_{3}(x)=\left|\varepsilon_{r 2}(x)-\varepsilon_{r 1}(x)\right|$ 


$$
\begin{gathered}
H_{1} \\
T_{3}(x) \underset{H_{0}}{\gtrless} \epsilon_{3} \\
H_{0}
\end{gathered}
$$

\subsubsection{Based on Impedance}

The null hypothesis is accepted if impedance of the first is closely similar to the second layer, $H_{0}: \eta_{2}(n)=\eta_{1}(n)$, otherwise the alternative is accepted if $H_{1}: \eta_{2}(n) \neq \eta_{1}(n)$. The test statistic in this case is $T_{4}(x)=\left|\eta_{r 2}(x)-\eta_{r 1}(x)\right|$

$$
\begin{gathered}
H_{1} \\
T_{4}(x) \underset{H_{0}}{\gtrless} \epsilon_{4}
\end{gathered}
$$

\subsection{Receiver Operating Characteristics}

Performance of detection and classification system shall be studies based on the receiver operating characteristics (ROC). ROC can be defined as the relationship between the true positive (hit), true negative (correct rejection), false positive (false alarm) and false negative (miss detection). Here, the analysis of the detection mechanism will be assessed in terms of true positives and miss detections are defined as:

$$
\begin{array}{r}
\text { hit }(\text { true detection })=\frac{P\left(H_{1}\right)}{P\left(H_{1}\right)} \quad \text { miss }(\text { flase rejection })=\frac{P\left(H_{0}\right)}{P\left(H_{1}\right)} \\
\text { true rejection }=\frac{P\left(H_{0}\right)}{P\left(H_{0}\right)} \quad \text { false alar } m=\frac{P\left(H_{1}\right)}{P\left(H_{0}\right)}
\end{array}
$$

Where, $P\left(H_{i}\right), i=0,1$ represents the probability of deciding the hypothesis 0 for no target and 1 for target presence. Three threshold levels are considered for each test statistic and the results are presented in section 6 .

\subsection{Target Detection and Parameter Estimation Procedure}

The GPR is configured to be operated as distance mode of collection with 1 pulse per millimeter setting and the data is imported to Matlab environment. The procedure for ground and target parameter estimation is summarized as follows:

Step 1 Call a DZT/DAT type file and reshape the GPR into Matlab environment $B s=$ fopen $($ 'filename.dat')\% calling into Matlab environment $B b s=$ fread $(B s$, 'uint 16')\% encoding with 16 bit (depends on the GPR type) $B$-scan $=$ reshape $(B b s, 512,[]) \%$ reshaping the row data into matrix form 
Step 2. For each A-scan, $A(t, x)$, subtract temporal mean to remove the DC bias or DC offset of the A-scam by subtracting the expected value of the A-scan, $u(t, x)=A(t, x)-E(A(x))$, where $E$ represents the expected value of the A-scan at position $x$.

Step 3. Consider a model signal in equation 1, and determine the first peak and peak time of the measurement A-scan at the vicinity of peak time of the model signal using equations 19 and 20, then estimate antenna height $h_{a}(x)$ using equation 21.

Step 4. Calculate air-ground reflection coefficient as a ratio of first peak to the incident voltage using equation 25 .

Step 5. Estimate ground surface impedance and relative permittivity with the assumption of lossless ground respectively using equation 26 and calculate mean square error (MSE) of the measured and estimated A-scans to validate the estimate using,

$$
M S E=\frac{\sum_{k=1}^{s p}\left(u_{m}(t)-u_{m}^{\prime}(t)\right)^{2}}{s p}
$$

Where, $s p$ is the number of samples per A-scan, which is based on the type of the GPR antenna.

Step 6. Layer reflection is estimated as maximum value of the difference of measured A-scan and modified model signal for time values higher than the first peak time plus half of the pulse width, and $k_{\alpha}$ is the peak amplitude ratio of the first layer and the reference using equations $26 \mathrm{a}$ and $26 b$.

$$
E_{r 2}=\max _{t}\left(x_{n}(t)-k_{\alpha} x_{m}\left(t-t_{\alpha}\right)\right), \quad t_{p 1}+\frac{\tau}{2} \leq t \leq M
$$

Step 6. Reflection coefficient of a layer as a function of the ratio of voltages and transmission products using equation 29.

Step 7. Estimate intrinsic impedance and relative permittivity of the second layer assuming lossless layer using equations 30 and 31 .

Step 8. Performance analysis of the detection model using ROC analysis using equation 36.

Step 9.Estimate antenna height (eq. 21) and target depth or thickness of the first layer is given as

$$
d_{1}(x)=\frac{c}{2 \sqrt{\varepsilon_{r 1}(x)}}\left(t_{p 2}(x)-t_{p 1}(x)\right)
$$

\section{SIMULATION}

\subsection{TL Modeling Simulation}

For the purpose of generating model signal, we consider the mixture model of water and clay soil (8, Helemi et al., 2008) and clay soil and target characteristics as in table 1. In the present case, 
relative permittivity considered for water is 81 and 2-6 for clay soil; and conductivities for clay is 0.05Siemens and 0.01 Siemens for water. In addition, relative permeability of all the materials is considered to be unity.

Table 1. Electrical properties at $100 \mathrm{MHz}$ and calculated parameters (Gebremichael, 2013).

\begin{tabular}{|llllll|}
\hline Medium & $\boldsymbol{\varepsilon}_{\boldsymbol{r}}$ & $\boldsymbol{\sigma}$ & $\boldsymbol{\alpha}$ & $\boldsymbol{\beta}$ & $\boldsymbol{Z}_{\boldsymbol{0}}$ \\
\hline Air & 1 & 0 & 0 & 20.98 & 376.6 \\
\hline Clay soil & $2-6$ & $0.01-0.1$ & $0.77-12$ & $29.7-54$ & $151-266$ \\
\hline Water & 81 & 0.01 & 0.209 & 188.61 & 41 \\
\hline TNT & 2.86 & 0.00029 & 0.03231 & 35.44 & 223 \\
\hline PVC & 3 & 0.003 & 0.3254 & 36.30 & 215 \\
\hline
\end{tabular}

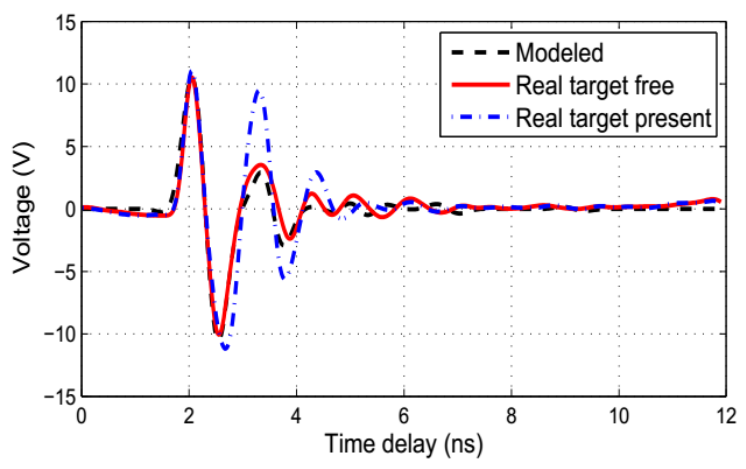

Figure 6. Comparison of Modeled A-scan and real GPR A-scan.

Figure 6 presents modeled A-scan of $15 \mathrm{GHz}$ GPR system for $10 \%$ moist, target free clay soil and practical A-scans for the same soil characteristics in the presence and absence of a target. Where, it can be seen from figure 6 that the target-free modeled A-scan is similar to the practical target free A-scan and the mean square is found to be 0.003 , which is acceptable for geophysical systems.

\subsection{Experimental Setup}

In the experiment, a Geophysical Survey Systems Inc. (GSSI) GPR antenna system with a center frequency of $1.5 \mathrm{GHz}$ and $80 \%$ bandwidth has been used. The GPR antenna is shielded, in which coupling between the transmitter and receiver antenna is negligible. The radar is adjusted in a distance mode of data collection with a survey wheel and 10 scans per $\mathrm{cm}, 16 \mathrm{bit}, 512$ sample points per scan and a range of $12 \mathrm{~ns}$. 
The GPR unit is suspended 1.5 to $3 \mathrm{~cm}$ above the ground surface. The targets were made of PVC cylinders of appropriate dimension and the cylinders were filled with wax and a small metal component at the center of the cylinder, which represent a plastic landmine with a firing pin. For the real data analysis, we consider the setup for three targets at different arrangements: PMN1 with dimensions $120 \times 50 \mathrm{~mm}$ buried at $42 \mathrm{~cm}$ and PMN2 with dimensions $110 \mathrm{~mm} \times 55 \mathrm{~mm}$ buried at $84 \mathrm{~cm}$ from the starting point of the scan in wet clay soil. The targets were buried at the same depth and oriented normal to the surface. Analysis results of the setup, with appropriate test statistics are shown in figures 7 to 10.

\section{RESULTS AND DISCUSSION}

\subsection{Peak Amplitude}

An EM wave to reflect from the second layer, there must be a dielectric contrast between the first and the second layers. If there is no difference in dielectric constant or the contrast is very low, which is due to inhomogeneity of the background soil, there is no reflection or the reflection is very weak. Figure 7 shows this scenario that, there is relatively high voltage amplitude at the target locations centered at $42 \mathrm{~cm} 84 \mathrm{~cm}$ and low reflection amplitude away from the target locations. The reflection amplitude of the first layer is due high due to that the contrast between the air and ground is high. Good detection could be achieved for threshold values between 2 and 6. Values less than 2 introduce false alarms whereas more than 6 lead to miss the target. The ROC analysis of this method is presented in table 2 for three threshold levels.

\subsection{Reflection Coefficient}

Uniformity of the reflection coefficient of the first boundary (between layers 1 and 2) implies that the ground surface is smooth and absence surface lying targets. However, high variation indicates the presence of surface lying target of roughness of the ground surface. Figure 8 shows the first boundary reflection is uniform. Positive reflection coefficient indicates the presence of air filled or void in the dielectric (Daniels, 2004), whereas negative coefficient indicates the presence of water field voids. Real data simulation results in figure 8 indicates that reflection coefficient of the first layer is almost smooth and reflection coefficient of the second layer is close to zero in the target free locations, whereas there is big negative reflection coefficients at the target locations. Classification would be easy if we use appropriate threshold lower than -0.1 and higher than -0.45 . 


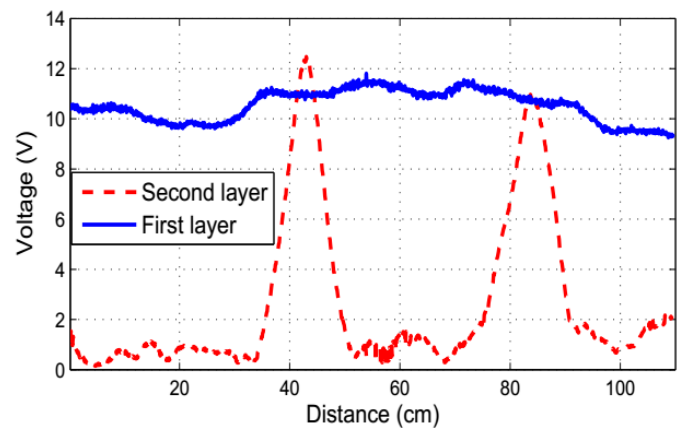

Figure 7. Reflected peak voltage amplitudes of the two layers.

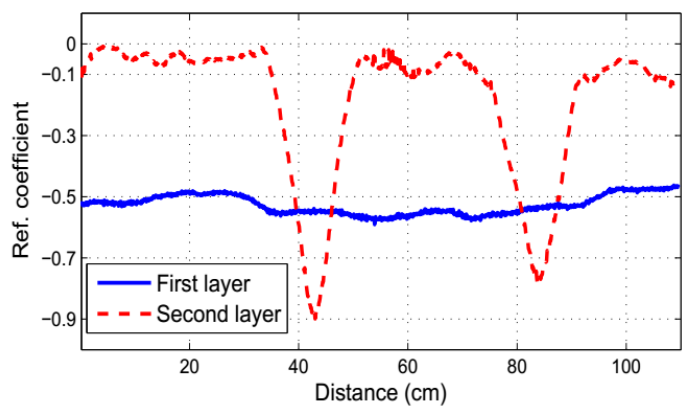

Figure 8. Reflection coefficients of first air-ground $\left(\Gamma_{01}\right)$ solid, and first-second layer $\left(\Gamma_{12}\right)$ broken.

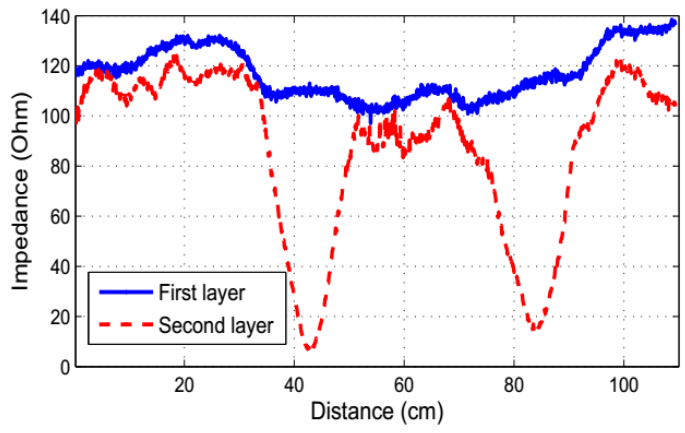

Figure 9. Intrinsic impedances of the first layer $\left(\eta_{1}\right)$ and second layer $\left(\eta_{2}\right)$.

\subsection{Impedance}

The impedance of second layer is greater than the first layer is similar to having a positive reflection coefficient in the first boundary. However, nearly equal impedance corresponds to having low reflection constant or similarly low voltage amplitude, and absence of a target. Real data simulation result in figure 9 shows that impedance of the ground surface lies between $110 \Omega$ and $140 \Omega$ which is realistic to the soil type under consideration. However, impedance of the second layer is close to the ground at the target free locations but very small, less than $20 \Omega$, at 
the center of the target location. Very good classification could be obtained for threshold values between $80 \Omega$ and $50 \Omega$ where diameter of the test statistic is close to the width of the landmine.

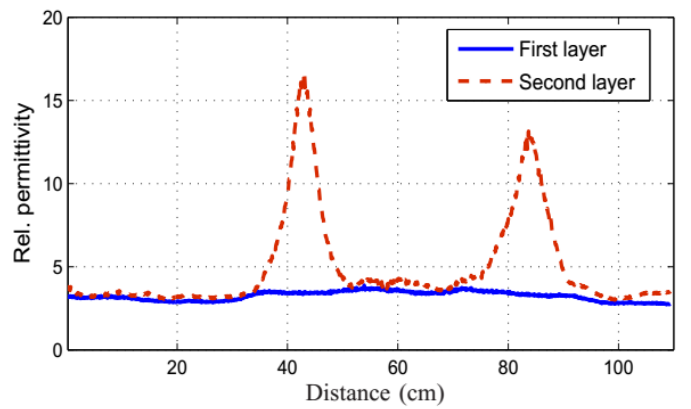

Figure 10. Relative permittivity of the first layer $\left(\varepsilon_{1}\right)$ and second layer $\left(\varepsilon_{2}\right)$, assuming loss less.

\subsection{Permittivity}

Assuming lossless layers means conductance of the soil is negligible, where the impedance of the medium is due to the permittivity and/or permeability components. Relative permittivity, $\varepsilon_{\mathrm{r}}$, of dry subsurface materials ranges between 3 and 6. Relative permittivity is always greater than unity and relative values away from the sub surface permittivity indicate the presence of a target. Common explosives, such TNT, RDX and Comp B have relative permittivity ranging 2.7 to 3.14, however, explosives like ammonium nitrate and nitroglycerin have very high value 7.10 and 19.00 respectively (Daniels, 2006). Simulation results in figure 10 indicates that permittivity values at the target free locations is exactly equal to the ground surface, however, large enough (close to 15) at the center of the target locations. Excellent classification could be achieved for threshold values ranging between 5 and 7.5 so that the width of the test statistic is close the diameter of the landmine.

Table 2. ROC analysis of the four methods.

\begin{tabular}{|c|c|c|c|c|c|}
\hline Method & $\begin{array}{l}\text { Threshold } \\
\text { level }\end{array}$ & $P\left(H_{l} / H_{l}\right)$ & $P\left(H_{0} / H_{0}\right)$ & $P\left(H_{l} / H_{0}\right)$ & $P\left(H_{0} / H_{l}\right)$ \\
\hline \multirow{3}{*}{$\begin{array}{l}\text { Peak } \\
\text { amplitude }\end{array}$} & $2.0 \mathrm{v}$ & 0.971 & 0.868 & 0.132 & 0.028 \\
\hline & $5.0 \mathrm{v}$ & 0.940 & 0.906 & 0.094 & 0.060 \\
\hline & $8.0 \mathrm{v}$ & 0.912 & 0.922 & 0.078 & 0.088 \\
\hline \multirow{3}{*}{$\begin{array}{l}\text { Reflection } \\
\text { coefficient }\end{array}$} & -0.10 & 0.962 & 0.866 & 0.134 & 0.074 \\
\hline & -0.30 & 0.931 & 0.908 & 0.092 & 0.068 \\
\hline & -0.50 & 0.905 & 0.922 & 0.078 & 0.096 \\
\hline \multirow{3}{*}{ Impedance } & $90 \Omega$ & 0.971 & 0.871 & 0.128 & 0.029 \\
\hline & $70 \Omega$ & 0.950 & 0.910 & 0.089 & 0.080 \\
\hline & $50 \Omega$ & 0.910 & 0.915 & 0.085 & 0.089 \\
\hline \multirow{3}{*}{ Permittivity } & 5.0 & 0.996 & 0.880 & 0.120 & 0.004 \\
\hline & 7.5 & 0.963 & 0.918 & 0.096 & 0.038 \\
\hline & 9.5 & 0.951 & 0.960 & 0.040 & 0.041 \\
\hline
\end{tabular}




\subsection{Arrival Time}

Arrival time can be used a means of estimating depth of the target and antenna height. The EM wave propagation velocity is inversely proportional to the permittivity of the medium and is assumed to be constant in a given medium. Depth is calculated as the product of the speed of the wave in a given medium and half of the two way travel time to be reflected back from the lower boundary of the medium estimated as:

$$
d(x)=\frac{c}{2 \sqrt{\varepsilon(x)}} t_{2}
$$

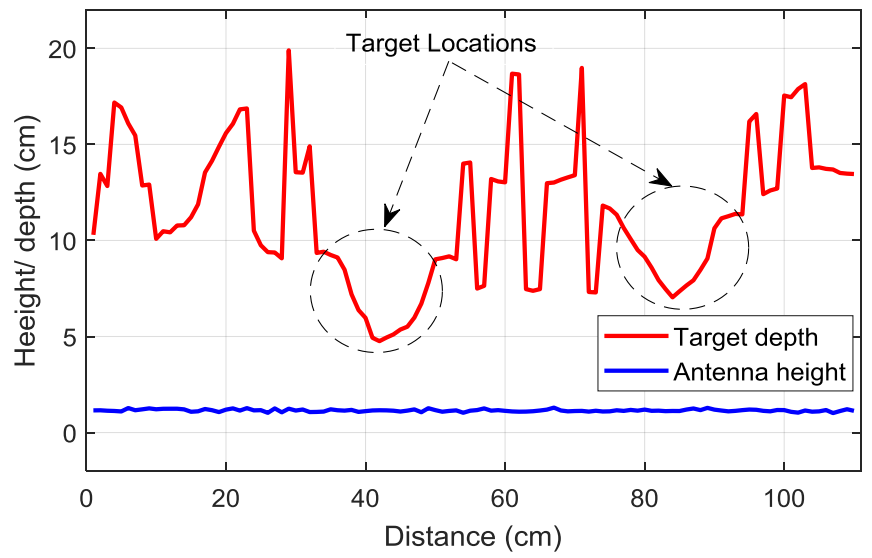

Figure 11. Antenna height and depth of the second layer.

Since, permittivity is used to detect the presence of a target, large two way travel time of the second layer is very large in the presence of a target. Anti-personal (AP) mines are buried shallowly in the top $10 \mathrm{~cm}$ and AT mines $30 \mathrm{~cm}$ deep in the ground soil. Simulation results in figure 11 shows that the arrival times of the first layer is similar, which indicates the absence of surface lying targets. Simulation results in figure 11 show that target depth is found to be below $10 \mathrm{~cm}$ and antenna height is about $2 \mathrm{~cm}$ from the surface.

\section{CONCLUSION}

The transmission line modeling approach is the best model for the study of GPR system Electromagnetic propagation. The reverse method is also useful to the parameter estimation of the subsurface and buried plastic landmines. The effectiveness of the methods is checked by the simulations presented in this paper. For known antenna height and magnitude of the incident voltage, the problem of parameter estimation can be simplified. Moreover, for known soil 
characteristics, nature of buried plastic landmine could be estimated perfectly using either one or fusion of the detection techniques presented in this paper.

All the hypotheses and analysis results indicate that the inverse transmission line modeling technique is the best method to estimate the subsurface parameters and detect the presence of buries objects. It has also shown that the four subsurface EM parameters convey the same information and equally apply if proper selection of the threshold is applied. Moreover, from the simulation results it is found that about $99.6 \%$ probability of detection is achieved at a cost of $12 \%$ false alarm. This is acceptable as UN accepted 99.6\% detection for humanitarian demining process. The antenna height and target depth are also found to be good estimates of the actual scenario. Finally, it can be concluded that the inverse TL modeling technique presented in this paper is possible to apply for wider applications for scanning subsurface, and study the structural status of buildings and bridges.

\section{ACKNOWLEDGENENTS}

This research was supported and funded by the Ethiopian Institute of Technology - Mekelle of Mekelle University, Technical University of Darmstadt and Ethiopian Engineering Capacity Building Program for the funding and scholarship. The data was collected at Griesheim old airport during my PhD study in 2011. I am very grateful Prof. Dr.-Ing. Zoubir, A. for providing the funding and technical guidance. Moreover, I am thankful to Mr. Tewodros Tesfaye for providing technical assistance during the data collection.

\section{REFERENCE}

Arunachalam, K., Melapundi, V., Udpa, L \& Udpa, S. 2006. Microwave NDT of Cement-based Materials Using Far-field Reflection Coefficients. NDT\&E International on Applications of Advanced Technology in Transportation, 39: 585-593.

Barkat, B., Zoubir, A. M \& Brown, C. L. 2000. Application of Time-Frequency Techniques for the Detection of Anti-Personnel Landmines. In: Proceeding of $10^{\text {th }}$ IEEE Signal Processing Workshop on Statistical Signal and Array Processing, (SSAP). Pennsylvania, USA, pp 494-597. 
Chien, P, K., Jing, Li., Yin, Wang., Huichun, Xing \& Ce, Richard Liu. 2007. Measurement of Layer Thickness and Permittivity Using New Multilayer Model From GPR Data. IEEE Transactions on Geoscience and Remote Sensing, 45: 2463-2470.

Daniels, D. J. 2004. Ground Penetrating Radar. $2^{\text {nd }}$ edition, ISBN: 0863413609, 9780863413605, The Institution of Electrical Engineers, 726p.

Daniels, D. J. 2006. A review of GPR for landmine detection, Sensing and Imaging. An International Journal, 7(3): 90-94.

GSSI. 2005. Handbook for GPR Inspection of Road Structures. Geophysical Survey Systems, Inc.

Helmi Z. M. S., Raja Abdullah, R.S.A., Mardeni, R \& Ratnasamy, M. 2008. Optimization of Ground Penetrating Radar (GPR) Mixture Model in Road Pavement Density Data Analysis, Geoscience and Remote Sensing Symposium, IEEE International, 3: 1326-1329

Jol, H. M. 2009. Ground Penetrating Radar Theory and Application. $1^{\text {st }}$ edition, ISBN: 978-0444-53348-7, Elsevier Science, 545p.

Lambot, S., Slob, E. C., van den Bosch, I., Stockbroeckx, B \& Vanclooster, M. 2004. Modeling of Ground-Penetrating Radar for Accurate Characterization of Subsurface Electric Properties. IEEE Transactions on Geoscience and Remote Sensing, 42(11): 398-404.

Lapinski, M. K. 2008, Multilayer Time Domain Transmission Line Model. $17^{\text {th }}$ International conference on Microwave, Radar and Wireless Communication (MIKON), pp. 1-3.

Liu, C.R., Li, J., Gan, X., Xing, H \& Chen, X. 2002. New model for estimating the thickness and permittivity of subsurface layers from GPR data. IEE Pro.-Radar Sonar Navigation, 149(6): 315-319.

MAction. 2009. Mine Action, Landmine Monitor, ISBN: 978-0-9738955-5-1, Special ten-Year review of the mine Ban treaty, Canada, pp 11-18.

MAction. 2010. Mine Action, Landmine Monitor, ISBN: 978-2-8399-1160-3, International Campaign to Ban Landmines Cluster Munition Coalition (ICBL-CMC), Canada, pp 1018.

MAction. 2011. Mine Action, Landmine Monitor, ISBN: 978-0-9738955-8-27-5, International Campaign to Ban Landmines Cluster Munition Coalition (ICBL-CMC), Canada, pp 3135 
MAction. 2014. Mine Action. Landmine Monitor, ISBN: 978-2-8399-1160-3, International Campaign to Ban Landmines Cluster Munition Coalition (ICBL-CMC), Canada, pp 1217

MAction. 2015. Mine Action. Landmine Monitor, ISBN: 978-2-8399-1707-0, International Campaign to Ban Landmines Cluster Munition Coalition (ICBL-CMC), Canada, pp1019.

Maurice, W. 2010. Electromagnetic Analysis Using Transmission Line Variables. ISBN 981-024428, World Scientific Publishing Co. Ltd., Singapore, pp 120-189.

Sadiku, M.N.O. 2010. Elements of Electromagnetics, ISBN: 0-19-513477-X, Oxford University Press, $537 \mathrm{p}$.

Gebremichael, G. T. 2013. Signal Processing Techniques for Landmine Detection Using Impulse Ground Penetrating Radar (ImGPR). PhD Thesis, pp 45-65 (available at http://tuprints.ulb.tu-darmstadt.de/3565/2/Gebremichael).

Valle, S., Zanzi, L \& Lenzi, G. 2000. 2D and 3D focusing of ground penetrating radar data for NDT. SPIE Proceedings of $8^{\text {th }}$ International Conference on Ground Penetrating Radar (GPR), pp 157-162.

Xu, X., Miller, E. L., Rappaport, C. M \& Sower, G. D. 2002. Statistical Method to Detect Subsurface Objects Using Array Ground-Penetrating Radar Data. IEEE Transactions on Geoscience and Remote Sensing, 40(4): 457-460.

Zoubir, A. M., Chant, I. J., Brown, C. L., Barkat, B \& Abeynayake, C. 2001. Signal Processing Techniques for Landmine Detection Using Impulse Ground Penetrating Radar. IEEE Sensors Journal, 2(1): 41-51. 